\title{
Protective effect of vascular endothelial growth factor against cardiopulmonary bypass-associated acute kidney injury in beagles
}

\author{
YIPING BAI ${ }^{1,2^{*}}$, YABING ZHANG $^{1 *}$, SHUTING YANG $^{3}$, MENGJUN WU $^{1}$, \\ YIBIN FANG ${ }^{2}$, JIANGUO FENG ${ }^{2}$ and BIN LIU ${ }^{1}$ \\ ${ }^{1}$ Department of Anesthesiology, West China Hospital of Sichuan University, Chengdu, Sichuan 610041; \\ ${ }^{2}$ Department of Anesthesiology, The Affiliated Hospital of Southwest Medical University, Luzhou, Sichuan 646000; \\ ${ }^{3}$ Department of Anesthesiology, The Second Affiliated Hospital of Sun Yat-Sen University, \\ Guangzhou, Guangdong 510085, P.R. China
}

Received June 25, 2017; Accepted November 3, 2017

DOI: $10.3892 / \mathrm{etm} .2017 .5460$

\begin{abstract}
The present study aimed to examine the hypothesis that vascular endothelial growth factor (VEGF) has a protective effect against cardiopulmonary bypass (CPB)-associated acute kidney injury (AKI). Eighteen male beagles were randomly allocated to three groups ( $n=6$ per group): Sham group, animals received sternotomy without going through CPB; CPB group, animals received CPB only; VEGF group, animals received CPB and VEGF. VEGF infusion was completed $1 \mathrm{~h}$ prior to the initiation of CPB. Renal microcirculation perfusion, serum creatinine (SCr) and blood urea nitrogen (BUN), histopathological injury score and apoptotic index were determined. Hypoxia inducible factor-1 $\alpha$, VEGF, phosphorylated (p)-Akt serine/threonine kinase (Akt), p-endothelial nitric oxide synthase (eNOS), cleaved caspase-3, B-cell lymphoma 2 (Bcl-2) and cluster of differentiation (CD)95 expression levels were assessed by western blot analysis, Enzyme-linked immunosorbent assay quantitative assays were used to evaluate tumor necrosis factor (TNF)- $\alpha$, interleukin (IL)-6, superoxide dismutase and malondialdehyde levels. Renal microcirculation perfusion of the VEGF group was higher than that of the CPB group $(\mathrm{P}<0.05)$ and lower than that of the sham surgery group $(\mathrm{P}<0.05)$. $\mathrm{SCr}$ and $\mathrm{BUN}$ were significantly elevated after $\mathrm{CPB}$ in the CPB and VEGF groups, with significantly lower levels in group VEGF than group CPB. Renal pathology scores and apoptotic indices were significantly lower in the VEGF group than the CPB group. Levels of TNF- $\alpha$, IL-6 in the VEGF group were significantly lower than in the CPB group. Levels of
\end{abstract}

Correspondence to: Dr Bin Liu, Department of Anesthesiology, West China Hospital of Sichuan University, 37 Wai Nan Guo Xue Xiang, Chengdu, Sichuan 610041, P.R. China

E-mail: liubinhxyy@163.com

${ }^{*}$ Contributed equally

Key words: acute kidney injury, renal blood flow, cardiopulmonary bypass, microcirculation, vascular endothelial growth factor
VEGF, p-Akt, p-eNOS and Bcl-2 expression in the VEGF group increased significantly in comparison with group CPB. Cleaved caspase-3 in the VEGF group was significantly lower than in the group CPB. CPB-associated reduction of renal microcirculation perfusion may predispose to AKI. VEGF appears to provide a protective effect on the kidneys through improvement in renal microperfusion.

\section{Introduction}

Postoperative acute kidney injury (AKI) is frequent after cardiac surgery in patients undergoing cardiopulmonary bypass (CPB) with a prevalence of around $20-30 \%$ in the adult population. It is associated with perioperative mortality as high as $20-80 \%$ (1-3). We $(4,5)$, as well as others (1) have previously demonstrated that a reduction in renal perfusion might be a contributing factor to postoperative kidney injury, however, there is a lack of an effective remedy to prevent the CPB-associated AKI. Goldberg and Dennen (6) found that AKI in the early stage can be fully recovered if early diagnosis and proper treatment are applied. Leonard et al (7) demonstrated that the reversal of renal dysfunction by targeted administration of vascular endothelial growth factor (VEGF) could be a novel potential therapeutic approach in ischemic renal disease. We hypothesize that VEGF has a role in protecting against $\mathrm{CPB}$-associated AKI by improving renal microperfusion.

\section{Materials and methods}

The animal protocol of the present study was approved by the Animal Care and Use Committee at West China Hospital (Chengdu, China) in accordance with the requirements of the Chinese Animal Care Committee.

All of the animals used in this study were treated in compliance with the Guide for the Care and Use of Laboratory Animals published by the US National Institutes of Health (NIH Publication, no. 85-23, revised 1996).

$C P B$ procedure. A total of 18 beagles, aged 2-4 years old, $10-15 \mathrm{~kg}$, were randomized into 3 groups. Sham group, received sternotomy and under general anesthesia for $5 \mathrm{~h}$; CPB group, 
$2 \mathrm{~h}$ of $\mathrm{CPB}, 2 \mathrm{~h}$ after CPB; VEGF group, $2 \mathrm{~h}$ of $\mathrm{CPB}, 2 \mathrm{~h}$ after CPB plus VEGF $0.1 \mathrm{mg} / \mathrm{kg}(8)$ intravenous administration in $1 \mathrm{~h}$ before $\mathrm{CPB}$.

A systemic CPB flow was established via aortic and venous cannulation in the right atrial appendage undergoing median sternotomy. CPB was performed for $2 \mathrm{~h}\left(32-34^{\circ} \mathrm{C}\right.$, $70-100 \mathrm{ml} / \mathrm{kg} / \mathrm{min}$ ) in the $2 \mathrm{~h}$ aortic cross clamping. The beagles were weaned from CPB, and received another $2 \mathrm{~h}$ observation. Core temperature, mean arterial pressure (MAP), arterial blood sample and ultrasound data were collected at five defined time-points $\left(T_{1-5}\right)$ (Fig. 1). $T_{1}$ was defined as initiation of CPB, $\mathrm{T}_{2}$ was defined as $1 \mathrm{~h}$ after initiation of CPB, $\mathrm{T}_{3}$ was defined as end of $\mathrm{CPB}, \mathrm{T}_{4}$ was defined as $1 \mathrm{~h}$ after $\mathrm{CPB}$, and $T_{5}$ was defined as $2 \mathrm{~h}$ after CPB. Kidneys were harvested at the end of the procedures for measurements.

Measurement of blood gas values. Arterial blood samples were collected in a polyethylene catheter from the left femoral artery. The blood gas parameters, including $\mathrm{pH}, \mathrm{PaO}_{2}, \mathrm{PaCO}_{2}$ and $\mathrm{Hb}$, were measured immediately with a blood gas analyzer (i-stat300G; Abbott Pharmaceutical Co. Ltd., Lake Bluff, IL, USA).

Contrast-enhanced ultrasound (CEU). After intravenous administration of stabilized sulfur hexafluoride microbubbles (SonoVue ${ }^{\circledR}$; Bracco, Milan, Italy), renal microcirculation was assessed by CEU (IU22; Philips Healthcare, Amsterdam, The Netherlands). Time-intensity curve (TIC) was derived from three region of interest (ROI) using QLAB post-analysis software. Parameters were calculated, including ascending slope rate (ASR), area under curve (AUC), peak intensity (DPI) and time to peak intensity (TTP). We averaged values from upper, middle and lower segments of kidney for anatomical factors.

Definition of AKI. AKI was defined by increase in serum creatinine ( $\mathrm{SCr}$ ) to $\geq 1.5$ times baseline according to Kidney Disease Improving Global Outcomes (KDIGO) guidelines (9). $\mathrm{SCr}$ and blood urea nitrogen (BUN) were evaluated before $\mathrm{CPB}$ and $2 \mathrm{~h}$ after $\mathrm{CPB}$.

Pathology scoring and determination of apoptosis rate. Specimens of right kidneys were collected at $2 \mathrm{~h}$ after $\mathrm{CPB}$, and were stored in formalin $4 \%$ for $24 \mathrm{~h}$. After washing, alcohol cleaning, and paraffin embedding slice, the slides were stained with hematoxylin and eosin (H\&E). The histological examination was blindly performed in three groups and 10 view fields per slide were examined. The severity of tissue damage was scored by two experienced doctors in accordance with the proportion of necrosis of renal tubule. Criteria: 0 , normal morphology; 1 , a small amount of renal tubular necrosis ( $\leq 10 \%) ; 2$, mild renal tubular necrosis $(10-25 \%)$; 3 , moderate renal tubular necrosis (26-75\%); and 4 , severe renal tubular necrosis $(\geq 75 \%)(10)$.

Paraffin sections were regularly dewaxed with xylene, prior to determination of the apoptosis rate by TdT-mediated dUTP nick end labeling (TUNEL).

Enzyme-linked immunosorbent assay (ELISA) and western blot analysis. Left kidneys were harvested at the end of the procedures, and immediately stored at $-80^{\circ} \mathrm{C}$ until assayed.
Tissues were ground with a mortar and pestle and then underwent repeated freeze-thaw cycles in lysis buffer (Promega, Madison, WI, USA). Tumor necrosis factor (TNF)- $\alpha$, MDA, SOD and interleukin (IL)-6 were measured via ELISA, and B-cell lymphoma 2 (Bcl-2), cluster of differentiation (CD)95, cleaved caspase-3, HIF-1 $\alpha$, VEGF, phosphorylated (p-)Akt and p-endothelial nitric oxide synthase (eNOS) protein levels were measured via western blot analysis.

Statistical analysis. Statistical analysis was performed using the SPSS 15.0 (SPSS, Inc., Chicago, IL, USA). Physiologic data were assessed for time effect and treatment using two-way repeated measurement ANOVA with Turkey post hoc tests, TIC Parameter, $\mathrm{SCr}$ and BUN levels, ELISA, western blot analysis were compared using a t-test. Values are shown as mean \pm standard deviation and $\mathrm{P}<0.05$ was considered significant.

\section{Results}

Basic characteristics. There were no differences in baseline values of $\mathrm{pH}, \mathrm{PaCO}_{2}, \mathrm{PaO}_{2}$ and $\mathrm{Hb}$ among groups. Core temperature (T) and MAP decreased $\mathrm{T}_{2}$ in comparison with baseline $(\mathrm{P}<0.05)$, with no significant difference after $\mathrm{CPB}$ (Fig. 1). $\mathrm{PaO}_{2}$ and $\mathrm{Hb}$ decreased significantly at $\mathrm{T}_{2-3}$, with $\mathrm{Hb}$ continually decreased at $\mathrm{T}_{4-5}(\mathrm{P}<0.05$; Fig. 1$)$.

TIC analysis of CEU. There were no differences in baseline values in the three groups. Compared with group sham, ASR decreased significantly at $T_{2-5}$, AUC and TTP increased significantly at $\mathrm{T}_{2-5}(\mathrm{P}<0.05)$ in CPB and VEGF group, with DPI decreased significantly at $\mathrm{T}_{3-5}$ in $\mathrm{CPB}$ group $(\mathrm{P}<0.05)$. Compared with the $\mathrm{T}_{1}$, ASR decreased and AUC, TTP increased significantly at $\mathrm{T}_{2-5}$ in $\mathrm{CPB}$ and VEGF group $(\mathrm{P}<0.05)$, with DPI decreased at $\mathrm{T}_{3-5}(\mathrm{P}<0.05)$ in $\mathrm{CPB}$ group. Compared with group CPB, ASR increased at $\mathrm{T}_{2-5}$, AUC and TTP decreased at $\mathrm{T}_{2-5}$ in VEGF group $(\mathrm{P}<0.05$; Fig. 2).

Renal function. Baseline values of SCr and BUN did not differ significantly among groups. After $2 \mathrm{~h} \mathrm{CPB}, \mathrm{SCr}$ and BUN increased significantly in $\mathrm{CPB}$ group in comparison with baseline, $\mathrm{SCr}$ and BUN in VEGF group were lower than that of CPB group. AKI occurred in 2 beagles in CPB group (1 beagle in stage 1,1 beagle in stage 2) and 1 beagle in VEGF group (in stage 1; Fig. 3).

\section{Histological analysis}

$H \& E$ staining. Damage scores of renal tubular epithelial cells in the CPB and VEGF groups were significantly higher than those in the sham group $(\mathrm{P}<0.05)$, and the score of the CPB group was higher than that of the VEGF group ( $\mathrm{P}<0.05$; Fig. 4$)$.

TUNEL staining. There were few apoptotic cells in the sham group. Apoptotic index in the CPB group was significantly higher than that in the VEGF group $(\mathrm{P}<0.05$; Fig. 4$)$.

Expression of TNF- $\alpha, I L-6, M D A$ and SOD. Levels of TNF- $\alpha$, IL-6 and MDA increased significantly in the CPB and VEGF groups compared with sham group, whereas SOD decreased significantly $(\mathrm{P}<0.05)$. TNF- $\alpha$ and IL-6 in the VEGF group decreased significantly compared with the CPB group (Fig. 5). 


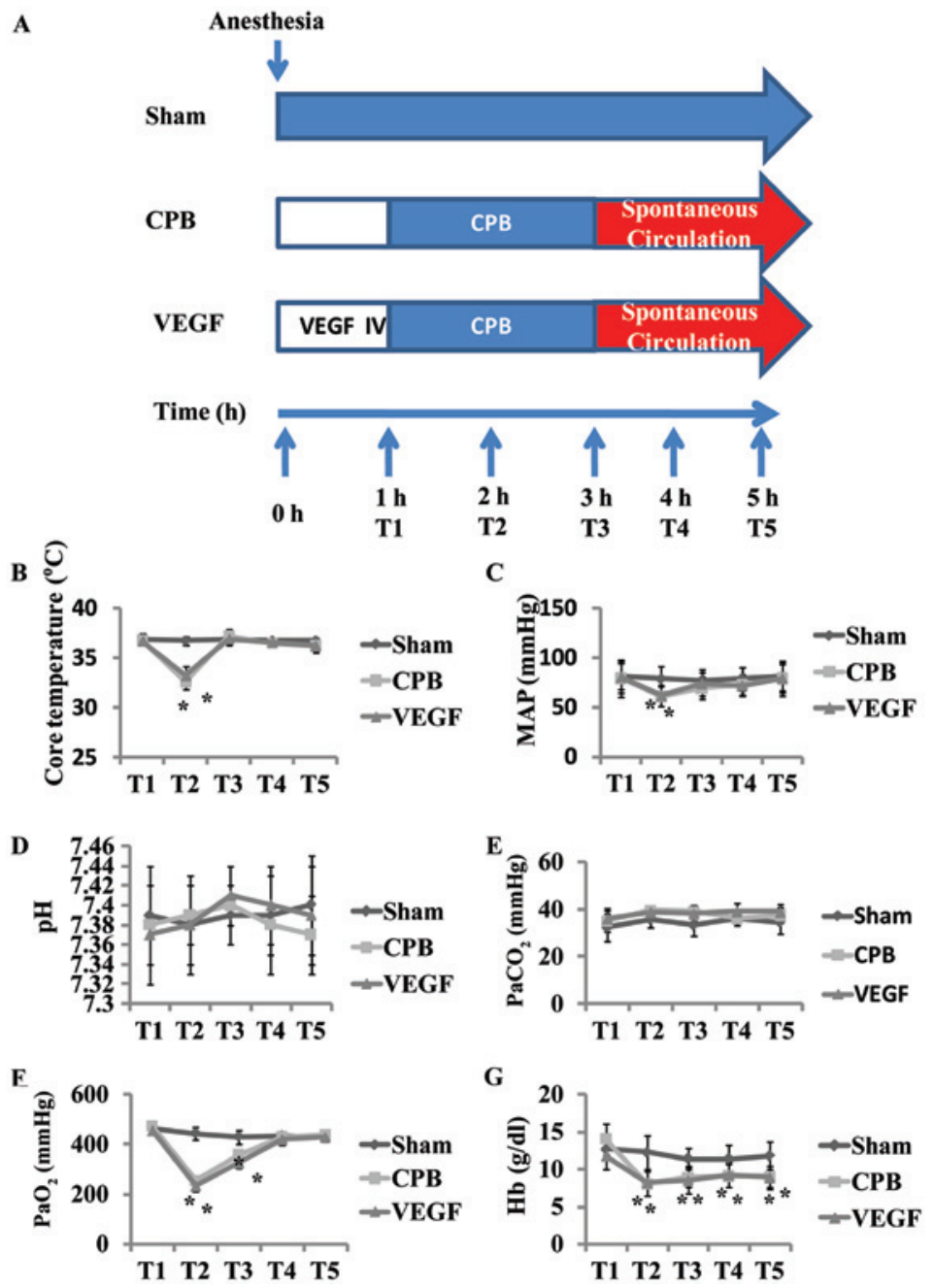

Figure 1. (A) Flow scheme of the animal experiment; (B) core temperature; (C) MAP and (D-G) blood gas values during the experiment. "P<0.05 vs. baseline. MAP, mean arterial pressure; CPB, cardiopulmonary bypass; VEGF, vascular endothelial growth factor.

Western blot analysis. Compared with the sham group, cleaved caspase-3, HIF-1 $\alpha$, VEGF, p-Akt and p-eNOS increased significantly in the CPB and VEGF groups, and $\mathrm{Bcl}-2$ decreased obviously $(\mathrm{P}<0.05)$. Compared with the CPB group, cleaved caspase-3 significantly decreased in the VEGF group $(\mathrm{P}<0.05)$, whereas Bcl-2, VEGF, $\mathrm{p}-\mathrm{Akt}$ and $\mathrm{p}-\mathrm{eNOS}$ increased significantly $(\mathrm{P}<0.05$; Fig. 6).

\section{Discussion}

Our previous studies have demonstrated that reduction in renal microcirculatory perfusion was associated with AKI even within normal range of blood pressure $(4,5)$, but we have no renal ultrasound data from patients during $\mathrm{CPB}$ to evaluate how renal microcirculatory perfusion effect on kidney for patients' safety consideration and surgeon's feeling. Kumar and Suneja (1) described that CPB-associated postoperative kidney injury was associated with renal microcirculatory and resorted to diagnostic technique and early diagnosis qualifying severity. CEU, from which decrease of the ASR and DPI and increase of AUC and TTP mean reduction of microperfusion, is able to quantify changes in renal microcirculation with advantages of real-time dynamic imaging, convenient, fast, no radiation, and no renal toxicity (11).
We found there were significant decreases in the ASR and DPI, increases in AUC and TTP at the beginning of CPB, at least from $1 \mathrm{~h}$ after initiation of $\mathrm{CPB}$, and throughout the whole procedure. It was suggestive of decreased renal microcirculation perfusion, and similar to our previous studies $(4,5)$, and confirmed by Andersson et al (12)and Pathi et al (13). By contrast, same tendency was found after VEGF administration, but there were much better outcomes, including ASR increased, AUC and TTP decreased significantly throughout the whole procedure in comparison with $\mathrm{CPB}$ group. Our data at least confirmed the existence of improved renal microcirculation after VEGF administration.

Since the target of the present study was to reduce AKI, the evaluation of a greater number of animals that develop AKI would be beneficial. However, our ischemic model was built via a CPB procedure for large animals, which was different from previous ischemic or metabolic kidney injury models created by clamping the organ arteries of small animals $(14,15)$. Clinical on-pump cardiac surgery was maximally stimulated in this study, so the incidence of AKI was not $100 \%$ in beagle models. AKI occurred in 2 beagles in the CPB group (33.3\%), which was close to previous reports $(20-30 \%)(1,3)$. One risk factor of AKI is ischemia reperfusion, so it is reasonable to think of increase of AKI incidence rate in the re-warming 

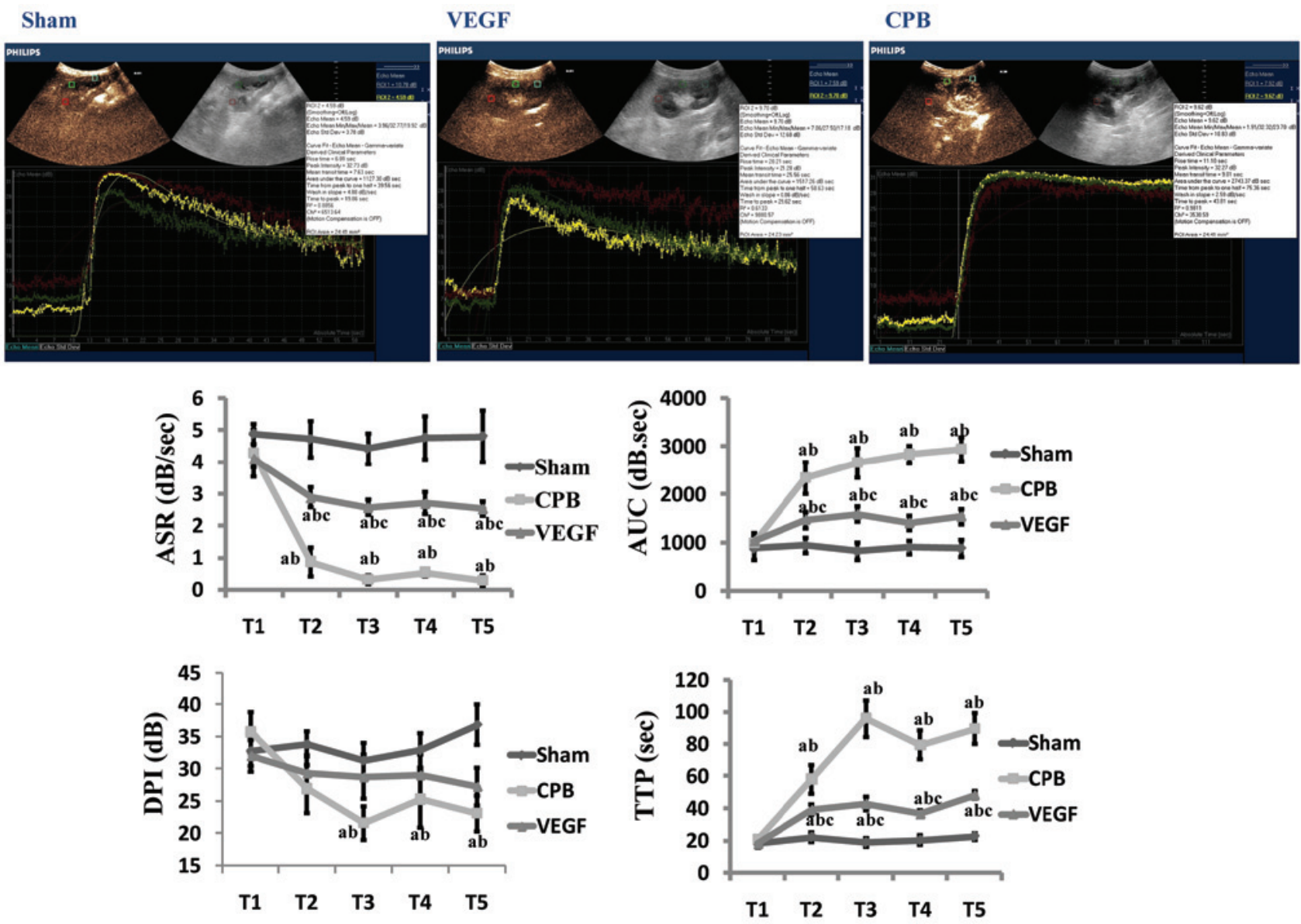

Figure 2. Image of time-intensity curve $2 \mathrm{~h}$ after $\mathrm{CPB}\left(\mathrm{T}_{5}\right)$, and time-intensity curve parameter analysis (ASR, AUC, DPI, and TTP). ${ }^{a} \mathrm{P}<0.05 \mathrm{vs}$. sham; ${ }^{\mathrm{b}} \mathrm{P}<0.05$ vs. $\mathrm{T}_{1} ;{ }^{\mathrm{C}} \mathrm{P}<0.05$ vs. CPB. CPB, cardiopulmonary bypass; VEGF, vascular endothelial growth factor; ASR, ascending slope rate; AUC, area under curve; DPI, peak intensity; TTP, time to peak intensity.
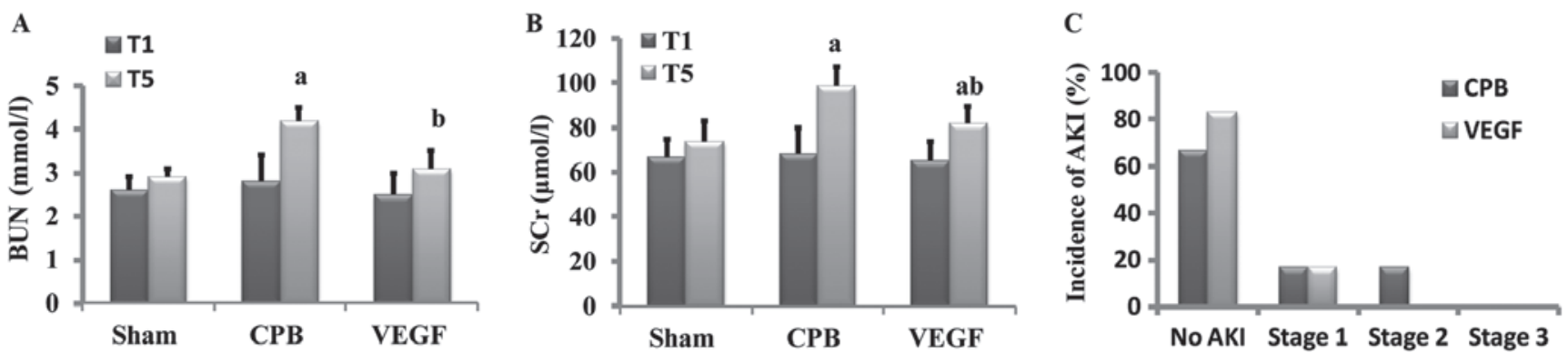

Figure 3. Levels of (A) BUN and (B) SCr in each group. (C) Incidence of AKI in CPB and VEGF groups; 2 occurred in the CPB group (33.3\%) and 1 in the VEGF group (16.7\%). Staging of AKI: Stage 1, 1.5-1.9 times baseline; stage 2, 2.0-2.9 times baseline; stage 3, 3.0 times baseline. ${ }^{a} \mathrm{P}<0.05$ vs. baseline, ${ }^{\text {b }} \mathrm{P}<0.05$ vs. CPB. BUN, blood urea nitrogen; SCr, serum creatinine; AKI, acute kidney injury; CPB, cardiopulmonary bypass; VEGF, vascular endothelial growth factor.

phase post-surgery, even if $2 \mathrm{~h}$ may be too short a time-frame to see significant effects in this study.

There were no instances of hypotension, severe anemia, and acid-base balance and water electrolyte abnormalities observed in the beagles during CPB, however, BUN and $\mathrm{SCr}$ increased significantly after $\mathrm{CPB}$. We also found more degeneration and necrosis from $\mathrm{H} \& \mathrm{E}$ staining slices and significantly increased apoptosis from TUNEL staining slices in comparison to sham group. Meanwhile, we observed that the changes were in consistence with CEU data, which was similar to the studies of Kumar and Suneja (1) and Iliescu et al (16) who concluded that damages of kidney endothelial microvascular system played an important role in the process of acute renal failure. Interestingly, BUN, SCr, degeneration and necrosis, and apoptosis were significantly improved after VEGF administration including less edema in renal tubules, reduction in renal tubular injury score, and decreased apoptosis. Thinking of renal microcirculation changes, it made us to recognize that VEGF may have protective effect via improvement of the renal microcirculation, which was not used as an angiogenic factor but some others.

This finding was similar to several recent studies which highlighted the important ability of VEGF in stabilization of the capillary structure (17-20). It has even been predicted 

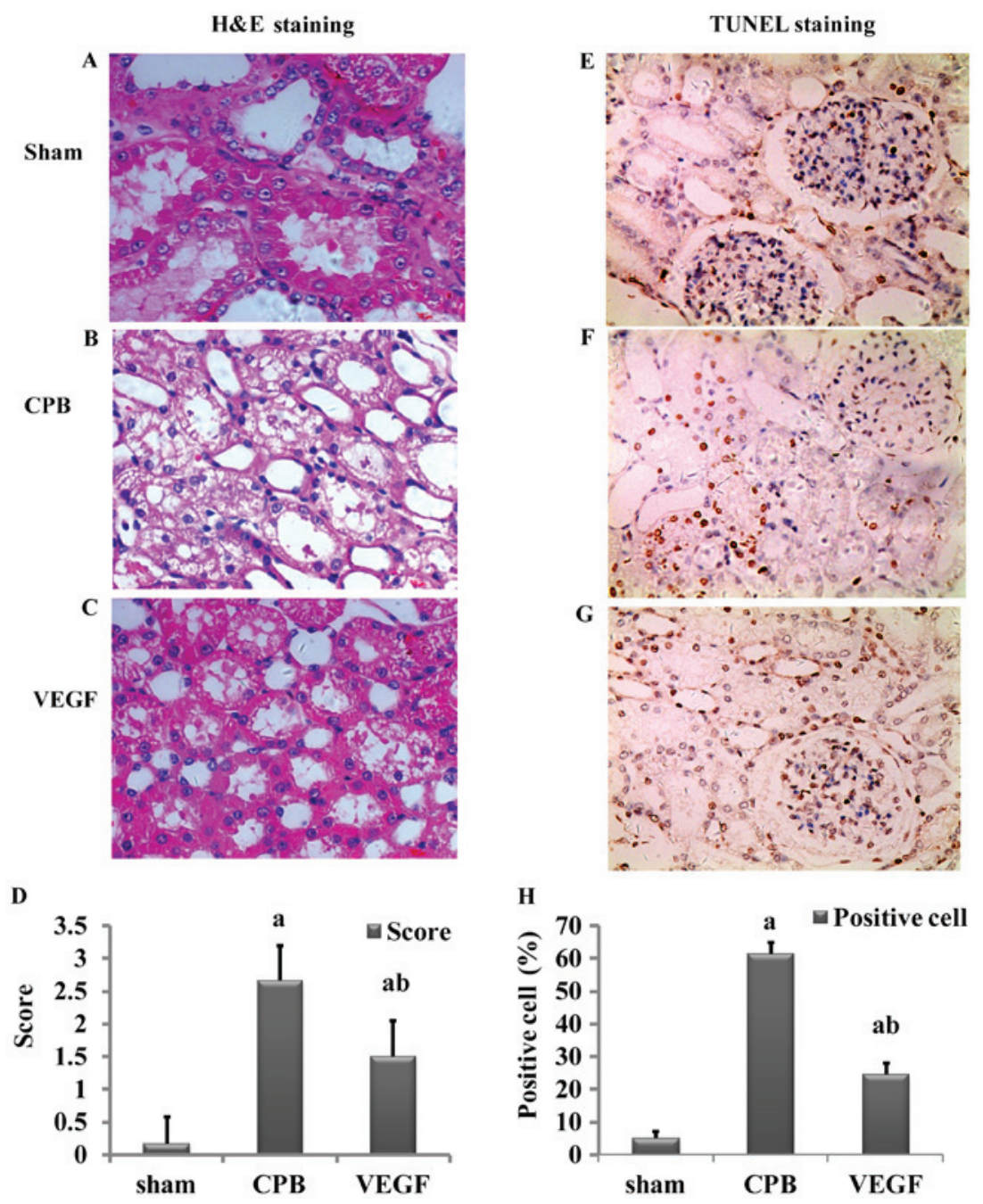

Figure 4. (A-C) H\&E staining; (D) AKI score; (E-G) TUNEL staining; and (H) percentage of apoptosis in the sham, CPB and VEGF groups. Necrotic tubules have necrotic shedding, edema and hyaline cast in H\&E staining. Apoptotic nuclei were pale yellow or brown, negative for blue, with a background color of white. Original magnification, $\mathrm{x} 400$. (D and H) ${ }^{\mathrm{a}} \mathrm{P}<0.05$ vs. sham; ${ }^{\mathrm{b}} \mathrm{P}<0.05$ vs. CPB. H\&E, hematoxylin and eosin; AKI, acute kidney injury; $\mathrm{CPB}$, cardiopulmonary bypass; VEGF, vascular endothelial growth factor.

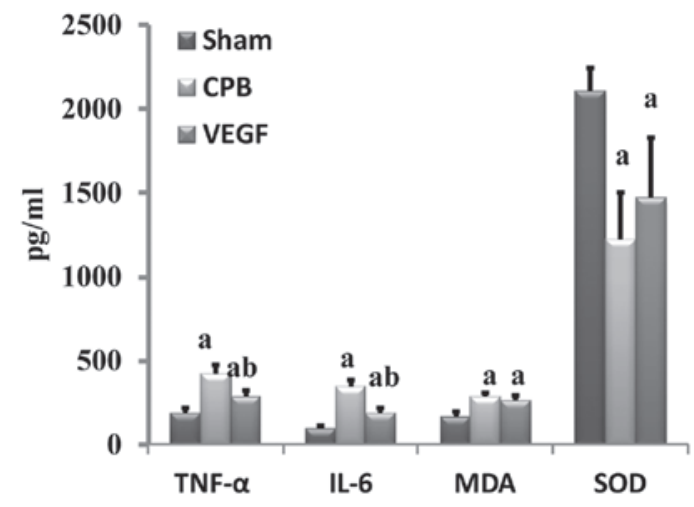

Figure 5. Expression of TNF- $\alpha$, IL-6, MDA and SOD. ${ }^{a} \mathrm{P}<0.05$ vs. sham, ${ }^{\mathrm{b}} \mathrm{P}<0.05$ vs. CPB. Data are expressed as mean \pm standard deviation. TNF, tumor necrosis factor; IL, interleukin; CPB, cardiopulmonary bypass; VEGF, vascular endothelial growth factor.

that VEGF could be a novel potential therapeutic approach in ischemic renal disease (21).

In further experiments on the relationship between $\mathrm{CPB}$ and renal microcirculation, we found that the expression of hypoxia-inducible factor-1 $\alpha$ (HIF-1 $\alpha$ ), which was upregulated in anoxic conditions $(22,23)$, increased after CPB under widely accepted normal blood pressure. It intuitively drove us to consider it a sign of renal ischemia. Meanwhile, the expression of VEGF was also elevated, the transcription and expression of which was induced by HIF-1 $\alpha$. VEGF mRNA expression was $4 \mathrm{~h}$ late after the increase of HIF-1 $\alpha(24,25)$. The increase of VEGF protein level may due to the release of synthesized VEGF. However, the increased concentration of VEGF in the CPB group was not enough to exert a positive effect on the renal capillaries, so we administered exogenous VEGF to beagles. At last, we obtained a significantly improved renal microcirculation and elevated expression of p-Akt and p-eNOS. P-Akt, which is activated by VEGF, could activate eNOS and increases the ratio of p-eNOS to total eNOS. P-eNOS induces nitric oxide (NO) (26), and increases the cGMP concentration that leads to vasodilatation by the activation of soluble guanylate cyclase, and improves microcirculation $(2,3)$. Besides the effect of vasodilatation, NO can be beneficial to inflammatory conditions via inhibiting the accumulation of neutrophils and leukocyte adhesion (27-29). We found inflammation, such as IL- 6 and TNF- $\alpha$, were lower 

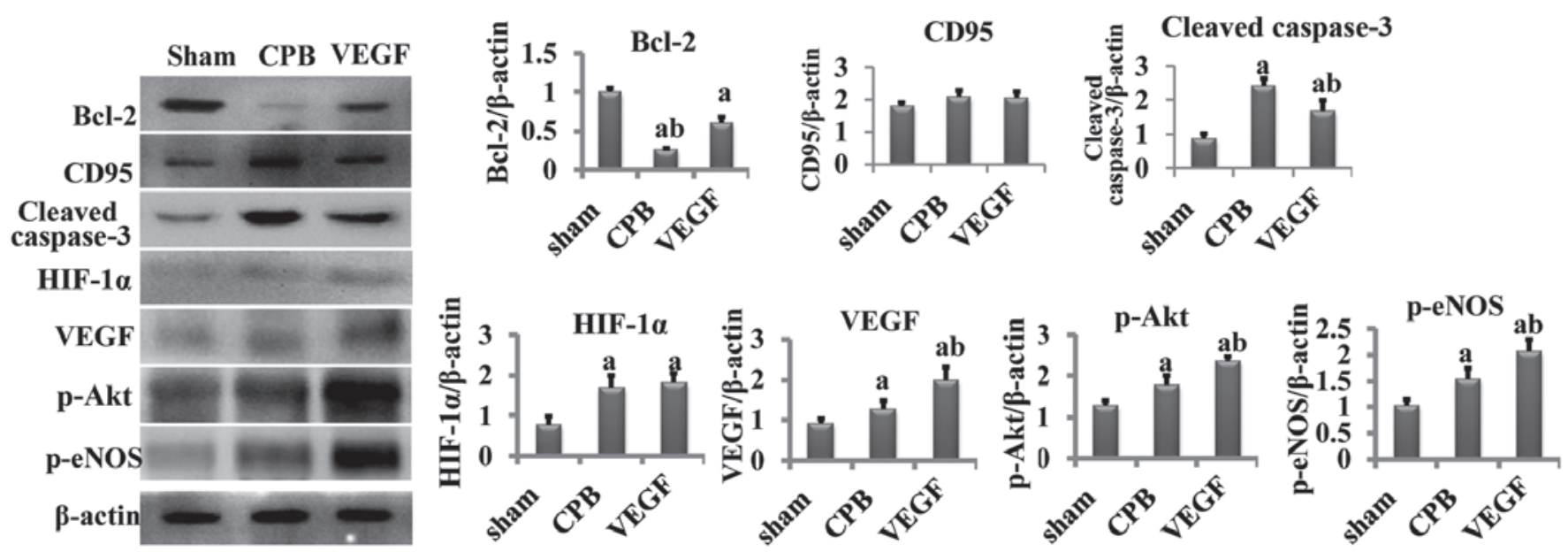

Figure 6. Concentration of Bcl-2, CD95, cleaved caspase-3, HIF-1 $\alpha$, VEGF, p-Akt, p-eNOS. ${ }^{a} \mathrm{P}<0.05$ vs. sham, ${ }^{b} \mathrm{P}<0.05$ vs. CPB. Bcl-2, B-cell lymphoma 2 ; $\mathrm{CD}$, cluster of differentiation; VEGF, vascular endothelial growth factor; p-eNOS, phosphorylated endothelial nitric oxide synthase; CPB, cardiopulmonary bypass.

after VEGF administration, which may be associated with anti-inflammatory effect of NO.

CPB-associated decrease of renal microperfusion, combined with ischemia reperfusion after $\mathrm{CPB}$, systemic inflammatory response, reduced postoperative cardiac output and use of vascular active drug, may contribute to renal microcirculatory injury (30). Renal microcirculation injury includes two stages: functional injury and structural injury (11,31). Function injury is at early stage, due to endothelial damage, inflammatory cytokines release, and decrease of expression of endothelial NO $(31,32)$. Elevated BUN and SCr quickly after CPB might be associated with functional renal injury $(6,33)$, which could be reversed by effective improvement of the microcirculation.

Tiny tweaks may lead to big changes to patients. We hope this study may provide important insights to support the use of CEU during CPB in clinical on-pump cardiac surgery, and initiate insights in renal microcirculation regimen for kidney protection.

In conclusion, $\mathrm{CPB}$-associated decrease of renal microcirculation perfusion may predispose to AKI. VEGF may produce protection against AKI through minimizing reduction in renal microperfusion.

\section{Acknowledgements}

This study was financially supported by nos. 2012FZ0121 and 81300110 .

\section{References}

1. Kumar AB and Suneja M: Cardiopulmonary bypass-associated acute kidney injury. Anesthesiology 114: 964-970, 2011.

2. Uchino S, Bellomo R, Goldsmith D, Bates S and Ronco S: An assessment of the RIFLE criteria for acute renal failure in hospitalized patients. Crit Care Med 34: 1913-1917, 2006.

3. Yang Y, Lang XB, Zhang P, Lv R, Wang YF and Chen JH: Remote ischemic preconditioning for prevention of acute kidney injury: A meta-analysis of randomized controlled trials. Am J Kidney Dis 64: 574-583, 2014.

4. Zhang L, Zhang JY, Zhong XF, Zhu D and Liu B: Exploration of renal microcirculation perfusion during cardiopulmonary bypass with contrast-enhanced ultrasound. Sichuan Da Xue Xue Bao Yi Xue Ban 46: 846-850, 2015 (In Chinese).
5. Zhong XF, Zhu D, Lu Q, Liu B and Peng YL: Monitoring renal microcirculation perfusion alteration with contrast-enhanced ultrasound during cardiopulmonary bypass. J Sichuan Univ Med Sci Edi 44: 646-650, 2013.

6. Goldberg R and Dennen P: Long-term outcomes of acute kidney injury. Adv Chronic Kidney Dis 15: 297-307, 2008.

7. Leonard EC, Friedrich JL and Basile DP: VEGF-121 preserves renal microvessel structure and ameliorates secondary renal disease following acute kidney injury. Am J Physiol Renal Physiol 295: F1648-F1657, 2008.

8. Masuda Y, Shimizu A, Mori T, Ishiwata T, Kitamura H, Ohashi R, Ishizaki M, Asano G, Sugisaki Y and Yamanaka N: Vascular endothelial growth factor enhances glomerular capillary repair and accelerates resolution of experimentally induced glomerulonephritis. Am J Pathol 159: 599-608, 2001.

9. Fadel FI, Abdel Rahman AM, Mohamed MF, Habib SA, Ibrahim MH, Sleem ZS, Bazaraa HM and Soliman MM: Plasma neutrophil gelatinase-associated lipocalin as an early biomarker for prediction of acute kidney injury after cardio-pulmonary bypass in pediatric cardiac surgery. Arch Med Sci 8: 250-255, 2012.

10. Badylak SF, Kern KB, Tacker WA, Ewy GA, Janas W and Carter A: The comparative pathology of open chest vs. mechanical closed chest cardiopulmonary resuscitation in dogs. Resuscitation 13: 249-264, 1986.

11. Gessner R and Dayton PA: Advances in molecular imaging with ultrasound. Mol Imaging 9: 117-127, 2010.

12. Andersson LG, Bratteby LE, Ekroth R, Hallhagen $\mathrm{S}$, Joachimsson PO, van der Linden J and Wesslén O: Renal function during cardiopulmonary bypass: Influence of pump flow and systemic blood pressure. Eur J Cardiothorac Surg 8: 597-602, 1994.

13. Pathi VL, Morrison J, MacPhaden A, Martin W, McQuiston AM and Wheatley DJ: Alterations in renal microcirculation during cardiopulmonary bypass. Ann Thorac Surg 65: 993-998, 1998.

14. Chade AR and Kelsen S: Renal microvascular disease determines the responses to revascularization in experimental renovascular disease. Circ Cardiovasc Interv 3: 376-383, 2010.

15. Chade AR, Krier JD, Textor SC, Lerman A and Lerman LO: Endothelin-a receptor blockade improves renal microvascular architecture and function in experimental hypercholesterolemia. J Am Soc Nephrol 17: 3394-3403, 2006.

16. Iliescu R, Fernandez SR, Kelsen S, Maric C and Chade AR: Role of renal microcirculation in experimental renovascular disease. Nephrol Dial Transplant 25: 1079-1087, 2010.

17. Chade AR and Kelsen S: Reversal of renal dysfunction by targeted administration of VEGF into the stenotic kidney: A novel potential therapeutic approach. Am J Physiol Renal Physiol 302: F1342-F1350, 2012.

18. Amadio M, Govoni S and Pascale A: Targeting VEGF in eye neovascularization: What's new?: A comprehensive review on current therapies and oligonucleotide-based interventions under development. Pharmacol Res 103: 253-269, 2016. 
19. Foster RR, Armstrong L, Baker S, Wong DW, Wylie EC, Ramnath R, Jenkins R, Singh A, Steadman R, Welsh GI, et al: Glycosaminoglycan regulation by VEGFA and VEGFC of the glomerular microvascular endothelial cell glycocalyx in vitro. Am J Pathol 183: 604-616, 2013.

20. Iruela-Arispe L, Gordon K, Hugo C, Duijvestijn AM, Claffey KP, Reilly M, Couser WG, Alpers CE and Johnson RJ: Participation of glomerular endothelial cells in the capillary repair of glomerulonephritis. Am J Pathol 147: 1715-1727, 1995.

21. Logue OC, McGowan JW, George EM and Bidwell GL III: Therapeutic angiogenesis by vascular endothelial growth factor supplementation for treatment of renal disease. Curr Opin Nephrol Hypertens 25: 404-409, 2016.

22. Larrivee B and Karsan A: Signaling pathways induced by vascular endothelial growth factor (Review). Int J Mol Med 5: 447-456, 2000

23. Hicklin DJ and Ellis LM: Role of the vascular endothelial growth factor pathway in tumor growth and angiogenesis. J Clin Oncol 23: 1011-1027, 2005

24. Lee YM, Jeong CH, Koo SY, Son MJ, Song HS, Bae SK, Raleigh JA, Chung HY, Yoo M and Kim KW: Determination of hypoxic region by hypoxia marker in developing mouse embryos in vivo: A possible signal for vessel development. Dev Dyn 220: $175-186,2001$

25. Vadlapatla RK, Vadlapudi AD and Mitra AK: Hypoxia-inducible factor-1 (HIF-1): A potential target for intervention in ocular neovascular diseases. Curr Drug Targets 14: 919-935, 2013.

26. Blanes MG, Oubaha M, Rautureau $Y$ and Gratton JP: Phosphorylation of tyrosine 801 of vascular endothelial growth factor receptor-2 is necessary for Akt-dependent endothelial nitric-oxide synthase activation and nitric oxide release from endothelial cells. J Biol Chem 282: 10660-10699, 2007.
27. Shu X, Keller TC IV, Begandt D, Butcher JT, Biwer L, Keller AS, Columbus L and Isakson BE: Endothelial nitric oxide synthase in the microcirculation. Cell Mol Life Sci 72: 4561-4575, 2015.

28. Kubes P, Suzuki M and Granger DN: Nitric oxide: An endogenous modulator of leukocyte adhesion. Proc Natl Acad Sci USA 88: 4651-5545, 1991.

29. Qin M,Landriscina A, Rosen JM, Wei G, Kao S, Olcott W, Agak GW, Paz KB, Bonventre J, Clendaniel A, et al: Nitric oxide-releasing nanoparticles prevent propionibacterium acnes-induced inflammation by both clearing the organism and inhibiting microbial stimulation of the innate immune response. J Invest Dermatol 135: 2723-2731, 2015.

30. Basile DP: Challenges of targeting vascular stability in acute kidney injury. Kidney Int 74: 257-258, 2008.

31. Garwood S: Cardiac surgery-associated acute renal injury: New paradigms and innovative therapies. J Cardiothorac Vasc Anesth 24: 990-1001, 2010.

32. Chade AR, Rodriguez-Porcel M, Grande JP, Krier JD, Lerman A, Romero JC, Napoli C and Lerman LO: Distinct renal injury in early atherosclerosis and renovascular disease. Circulation 106: 1165-1171, 2002.

33. Badal SS and Danesh FR: New insights into molecular mechanisms of diabetic kidney disease. Am J Kidney Dis 63 (2 Suppl 2): S63-S83, 2014 International (CC BY-NC-ND 4.0) License. 